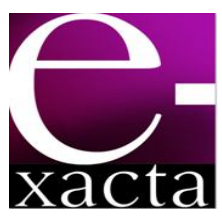

ISSN: 1984-3151

\section{RECONHECIMENTO DE AROMAS E APLICAÇÃO DE TESTES AFETIVOS COMO FORMA DE APRENDIZADO}

\author{
RECOGNITION OF AROMA AND APPLICATION TESTING AS A MEANS OF
} LEARNING AFFECTIVE

\author{
Kamila de Oliveira do Nascimento'; Dilson Fagundes Ribeiro; Elga Batista ${ }^{3}$ \\ 1 Mestre em Ciência e Tecnologia de Alimentos. 2008. DTA/UFRRJ. \\ kamila.nascimento@yahoo.com.br \\ 2 Mestre em Ciência e Tecnologia de Alimentos. 2012. DTA/UFRRJ. \\ Professor do Instituto Federal de Educação, Ciência e Tecnologia do \\ Espírito Santo. dilson-fagundes@hotmail.com \\ 3 Mestre em Ciência e Tecnologia de Alimentos. 2008. DTA/UFRRJ. \\ Professora do Centro Universitário Augusto Motta. Bonsucesso, RJ. \\ elga.silva@hotmail.com
}

Recebido em: 03/04/2014 - Aprovado em: 30/04/2014 - Disponibilizado em: 31/05/2014

\begin{abstract}
RESUMO: A análise sensorial pode ser utilizada para se evocar, medir, analisar e interpretar as reações dos consumidores em relação às características dos alimentos. Nesse contexto, os atributos olfativos são importantes para a escolha de um alimento. O objetivo deste trabalho foi de verificar a percepção sensorial das alunas do curso de pós-graduação na área de Nutrição, com uma abordagem didática aplicada pelo reconhecimento de aromas e aplicação de testes afetivos, como forma de aprendizado. Para a realização das análises, 30 alunas avaliaram 16 amostras (café, canela, cravo, vinagre, cachaça, azeite, orégano, molho shoyo, xarope de guaraná, açúcar, curry, pimenta calabresa, queijo parmesão, limão, vinho tinto e coentro). Também foram realizados os testes de preferência e intenção de compra de duas marcas de biscoitos amanteigados. Os resultados mostraram que os maiores índices de acertos foram verificados para os aromas das amostras de limão (84\%), café (80\%), canela (80\%), cravo (80\%) e vinagre (80\%); e que a pimenta calabresa e o coentro não foram reconhecidos por nenhum provador. Além disso, também foi observado que os biscoitos amanteigados da marca A apresentaram maior aceitabilidade, quando $24 \%$ dos participantes afirmaram que certamente comprariam o produto. A partir dos resultados observados, foi possível verificar que as práticas realizadas podem contribuir para que o aluno exercite sua capacidade de perceber diferenças entre aromas de diferentes amostras de alimento e bebidas. Portanto, pode-se verificar a importância da análise sensorial como ciência, contribuindo de forma didática no aprendizado dos alunos do curso de Nutrição.
\end{abstract}

PALAVRAS-CHAVE: Análise sensorial. Alimentos. Preferência.

ABSTRACT: Sensory analysis can be used to evoke, measure, analyze and interpret the reactions of consumers in relation to food characteristics. In this context, the olfactory attributes are important for choosing a food. The objective of this work was to verify the perception of Nutrition's students, with a teaching approach applied by the recognition of aromas and applying affective tests as a way of learning. For the analyzes, 30 students evaluated 16 samples (coffee, cinnamon, cloves, vinegar, rum, olive oil, oregano, soy sauce, guarana syrup, sugar, curry, chilli, parmesan cheese, lemon, red wine and coriander). Tests of preference and intention to purchase two brands of butter cookies were also conducted. The results showed that the highest rate of correct answers were checked for the aromas of lemon samples (84\%), coffee (80\%), cinnamon (80\%), clove (80\%) and vinegar (80\%) and pepper Calabrese and coriander were not recognized by any tester. Furthermore, it was also observed that butter cookies brand $A$ had higher acceptability, where $24 \%$ of participants said they certainly would buy the product. From the observed results it was verified that the practices performed may contribute to the student to exercise your ability to 
perceive differences between scents from different samples of food and beverages. Therefore, it's possible to see the sensory analysis importance's as a science, contributing didactically in the students of Nutrition learning.

KEYWORDS: Sensory analysis. Food. Preference.

\section{INTRODUÇÃO}

A análise sensorial pode ser definida como disciplina científica utilizada para se evocar, medir, analisar e interpretar reações das características dos alimentos e materiais da forma como são percebidas pelos sistemas sensoriais olfativo, gustativo, tátil, auditivo e visual, que avaliam os atributos dos alimentos (TEIXEIRA, 2009).

A qualidade sensorial pode ser determinada pela interação entre o homem e o alimento e envolve aspectos culturais, étnicos e sociológicos (SIMÕES; WASZCYNSKYJ; WOSIACKI, 2009). Essa qualidade está associada aos estímulos provenientes dos alimentos e das condições fisiológicas e sociológicas dos indivíduos que os avaliam dentro de um contexto ambiental em que se localizam 0 produto e 0 indivíduo (MINIM, 2006).

Ainda segundo Minim (2006), a qualidade sensorial de um alimento pode variar de pessoa para pessoa e ser influenciada por fatores característicos dos alimentos, como aparência, sabor, textura, forma, método de preparo, custo e sazonalidade. Além desses aspectos, existem ainda influências relativas aos fatores característicos do indivíduo, como idade, sexo, grau de educação, cultura, estado fisiológico e renda.

Os atributos sensoriais (sabor, aroma, textura ou consistência e aparência) são determinantes para o consumo do alimento, bem como para a saciedade, ingestão e seleção do alimento numa refeição (ELMAN; SILVA, 2006).

O apreço por aromas não é um interesse recente do homem, visto que relatos históricos afirmam que desde a época do antigo Egito já eram empregados especiarias e seus derivados no preparo dos alimentos há milhares de anos (TRAJANO et al., 2009).

O olfato é o sentido que permite identificar-se o odor e o aroma do produto, sendo que o odor é percebido pelo órgão olfativo quando substâncias voláteis são aspiradas (ARAÚJO et al., 2011).

Já o aroma é a propriedade sensorial perceptível pelo órgão olfativo via retronasal. Através da degustação, compostos voláteis se desprendem do alimento e ativam os receptores no epitélio olfativo, localizado no dorso da cavidade nasal, no septo e nos turbinados superiores, estruturas ósseas que criam um fluxo aéreo turbulento, que permite a percepção dos compostos voláteis pelas células olfativas (ARAÚJO et al., 2011).

Assim, o aroma dos produtos alimentícios constitui um dos principais impulsionadores da aceitação pelos consumidores. Sua caracterização representa, portanto, um grande desafio para a indústria de alimentos (PARAVISINI et al., 2014).

O aumento da concorrência, as novas oportunidades estimuladas pelo livre comércio e expansão dos mercados mundiais aceleraram consideravelmente a exigência mundial da indústria de alimentos para novos produtos. Assim, estudar a aceitação de produtos alimentícios pelos consumidores tem sido benéfica em vários aspectos, o que estimula o interesse de disciplinas fora da ciência dos alimentos e serve como um mecanismo adicional para avaliação sensorial, visando estabelecer-se como fonte de informação (SIDEL; STONE, 1993).

Desse modo, a análise sensorial tem se mostrado uma ferramenta muito importante e útil na avaliação 
das características dos produtos, possibilitando, dessa maneira, medir-se a qualidade do alimento e de suas matérias primas.

Assim, realizar atividades práticas no ensino superior representa uma alternativa interessante para fomentar, junto aos alunos, formas de aplicar os conhecimentos obtidos a partir de aulas expositivas. Diversas metodologias podem ser empregadas na área de Nutrição para alcançar se esse objetivo, entre elas aquelas inerentes aos conteúdos de disciplinas da área de Ciência e Tecnologia dos Alimentos.

Diante do exposto, objetivou-se com este trabalho verificar a percepção sensorial dos alunos do curso de pós-graduação na área de Nutrição, com uma abordagem didática aplicada pelo reconhecimento de aromas e aplicação de testes afetivos, como forma de aprendizado.

\section{MATERIAIS E MÉTODOS}

\subsection{TESTES DE RECONHECIMENTO DE AROMAS}

O painel sensorial foi composto por trinta alunos do curso de pós-graduação na área de Nutrição de uma Universidade do Rio de Janeiro. Esses indivíduos foram submetidos a 16 estímulos olfativos, apresentados de forma casualizada, aleatória e sem nenhuma sugestão quanto à sua identificação. $O$ teste foi conduzido no mês de julho de 2012.

Os provadores foram instruídos a cheirar as amostras com intervalos de vinte segundos entre uma amostra e outra. Logo que cheiravam, descreviam na ficha os odores percebidos ao lado de cada código. Para amenizar o cansaço olfativo, foram orientados a cheirar a pele do próprio pulso, visando também a neutralizar o aroma anterior. As dezesseis amostras utilizadas para provocar os estímulos foram: café, canela, cravo, vinagre, cachaça, azeite, orégano, molho shoyo, xarope de guaraná, açúcar, curry, pimenta calabresa, queijo parmesão, limão, vinho tinto e coentro. As julgadoras não receberam quaisquer treinamentos prévios à execução da análise e foram instruídas sobre os procedimentos para a sua realização momentos antes da execução do teste (DOTY; SHAMAN; DANN, 1984; INSTITUTO ADOLFO LUTZ, 2008).

\subsection{Testes de Preferência e InTenção de COMPRAS DOS BISCOITOS AMANTEIGADOS}

Os testes foram realizados no mês de agosto de 2012 com duas marcas comerciais de diferentes biscoitos amanteigados foram adquiridas no comércio local do Rio de Janeiro. A aparência de cada formulação foi julgada em blocos completos, casualizados, e as características de sabor e textura de forma monádica. Os provadores avaliaram as formulações em cabines individuais, onde os biscoitos foram dispostos em pratos de fundo branco, codificados com número de três dígitos, iluminados por luz natural do dia.

Dois testes afetivos foram conduzidos, sendo um de aceitação (STONE; SIDEL, 1993) e de intenção de compra (SCHUTZ, 1965). O painel sensorial foi composto por 30 julgadores não treinados. Para o teste afetivo de aceitação, foi utilizada uma escala hedônica estruturada de nove pontos ("gostei muitíssimo" a "desgostei muitíssimo") para avaliação do atributo sabor e textura. $\mathrm{Na}$ análise de intenção de compra, foi empregada uma escala de 5 pontos com impressões compreendidas entre "certamente compraria" e "certamente não compraria".

\section{ANÁlises ESTATÍSTICAS}

Os resultados foram verificados por análise de variância (ANOVA) e teste de Tukey em nível de 5\% de significância, para comparação das médias. 


\section{Resultados e discussões}

Pela Figura 1, observam-se os resultados para a avaliação do teste de reconhecimento de aromas.

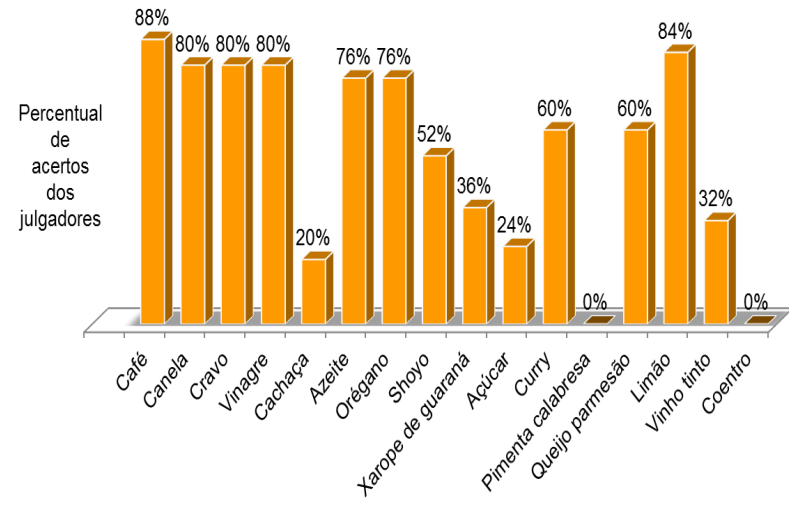

Figura 1. Percentual de acertos pelos provadores no teste de reconhecimento de aromas

De acordo com a Figura 1, verifica-se que $68 \%, 76 \%$ e $80 \%$, respectivamente, não conseguiram identificar corretamente os aromas do vinho tinto, açúcar e cachaça. Além disso, também é possível observar que nenhum dos provadores foi capaz de identificar as amostras de pimenta calabresa e coentro.

Os maiores percentuais de associação correta entre aroma e amostra foram verificados nos testes conduzidos com limão (84\%), café $(80 \%)$, canela $(80 \%)$, cravo $(80 \%)$ e vinagre $(80 \%)$. Esse resultado pode ser justificado pela ampla utilização desses alimentos na culinária brasileira, bem como pelos aromas intensos percebidos a partir dessas amostras.

Segundo Tempère et al. (2014), poucos estudos têm se centrado na percepção olfativa de profissionais do vinho. No entanto, observam-se variações consideráveis na sensibilidade olfativa dos peritos aos compostos do vinho, incluindo etilfenóis. Estudo realizado por Tempère et al. (2011) revelou que 1\% dos 134 especialistas testados apresentaram hiposmia (perda parcial da capacidade olfativa) específica para etilfenóis. Esse déficit sensorial específico certamente afeta a capacidade de identificação no rico ambiente odoríferos de vinho.

De acordo com Goyert et al. (2007), 22\% dos provadores não conseguiram identificar corretamente o aroma de baunilha, enquanto, para os aromas de álcool e hortelã, verificou-se uma redução na identificação de apenas $8 \%$. Esses autores sugerem que a potência trigeminal pode, em parte, explicar essas diferenças adaptativas.

O aumento ou a diminuição de sensibilidade também pode resultar da integração sensorial multimodal dos sistemas neurais olfativos e gustativos (ADHIKARI et al., 2006).

O nariz humano contém mais de 100 milhões de receptores especializados na identificação das moléculas de odores, que possuem formas e tamanhos específicos. A camada de muco dissolve essas moléculas assim que elas chegam aos receptores. O cérebro, por sua vez, é capaz de interpretar e identificar os diferentes tipos de odores (DE MELO LISBOA; PAGE; GUY, 2009).

A adaptação e recuperação da adaptação fundamentalmente influenciam a forma de como detectar os odores e responder ao ambiente. Entender os mecanismos subjacentes à adaptação de odor em humanos requer uma primeira compreensão de como diferentes odores interagem para ativar o processo de adaptação. A natureza complexa do sistema olfativo e a subjetividade da resposta humana resultaram em numerosos estudos sobre a medição de materiais odoríferos por meios químicos e físicos (STONE; PANGBORN; OUGH, 1965).

O olfato é um sentido primordial de enorme significado para os seres humanos e animais. Sabese que uma alteração sutil na estrutura de odores químicos altera drasticamente o seu odor percebido. Neurônios sensoriais olfativos que detectam odores expressam diferentes receptores, provocando 
diferentes sinais no cérebro e, assim, geram percepções de odores distintos (KRANC, 2013).

Os resultados das avaliações dos provadores das amostras de biscoitos estão apresentados na Tabela 1.

\section{Tabela 1}

Resultado da avaliação estatística dos testes de preferência referentes aos atributos dos biscoitos amanteigados.

\begin{tabular}{lccc}
\hline Atributos & \multicolumn{2}{c}{ Amostras } & *DMS \\
\hline Aparência & A & B & \\
Sabor & $8,00^{\mathrm{a}}$ & $8,06^{\mathrm{a}}$ & 0,62 \\
Textura & $7,26^{\mathrm{a}}$ & $4,4^{\mathrm{b}}$ & 0,57 \\
& $7,00^{\mathrm{a}}$ & $4,2^{\mathrm{b}}$ & 0,55 \\
\hline
\end{tabular}

Letras diferentes na mesma linha apresentam diferença significativa $(p \leq 0,05)$ pelo Teste de Tukey; ${ }^{* *} \mathrm{DMS}$ : Diferença Mínima Significativa.

Verifica-se, pela Tabela 1, que as amostras comerciais de biscoitos não apresentaram diferenças significativas $(p \leq 0,05)$ entre elas em relação à aparência. Entretanto, observa-se que a amostra $A$ apresentou um sabor e textura melhores que a amostra B, mostrando diferenças significativas $(p \leq 0,05)$.

Apesar de a amostra A ter sido a preferida, os provadores consideraram que ambas as amostras apresentaram uma textura mais resistente ao morder, sendo a amostra A aquela a apresentar maior crocância. Observa-se que, em relação ao sabor a amostra B, aquela foi considerada levemente mais doce pelos julgadores. Adicionalmente, o painel sensorial também afirmou ter percebido um "gosto acentuado de ovo cru", o que poderia justificar a avaliação "desgostei levemente", uma vez que esses fatores podem ter contribuído para as menores notas dadas a este produto.

Segundo Altisent et al. (2013), o sabor continua sendo um motivo dominante no momento em que os consumidores optam por adquirir um produto. A avaliação sensorial dos atributos de textura também é a principal maneira pela qual os consumidores julgam a qualidade de muitos produtos alimentares (PEREIRA; BENNETT; LUCKMAN, 2005). Assim, quando o sabor e textura são considerados aceitáveis, novas compras são estimuladas, mesmo que a aparência não seja a ideal do ponto de vista do consumidor. No entanto, o que se considera ser aceitável varia entre os indivíduos, grupos de consumidores e regiões geográficas (SEPPÄ et al., 2013).

Pela Figura 2, observa-se a intenção de compra em relação a duas marcas de biscoitos comerciais.

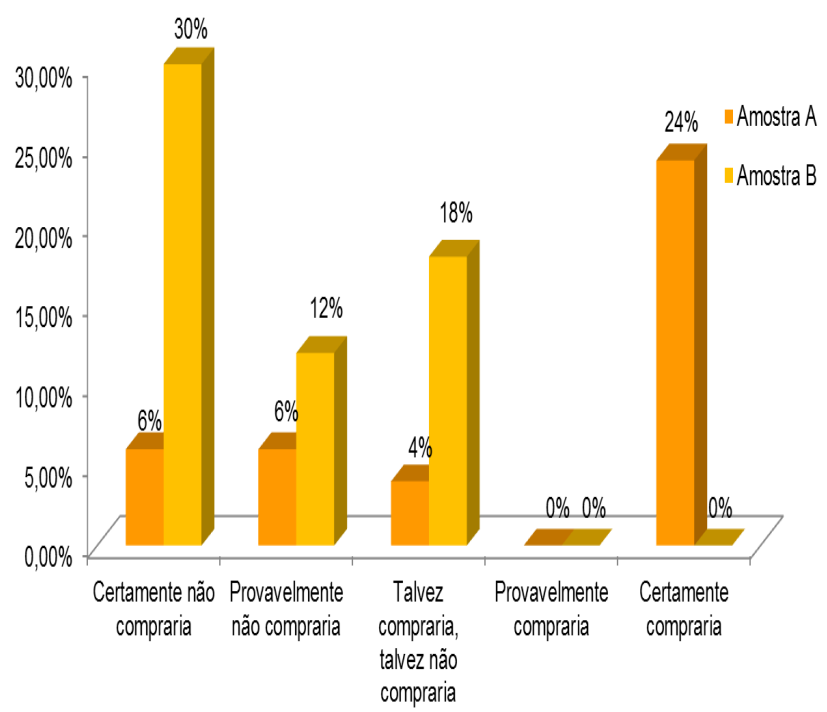

Figura 2. Intenç̧ão de compra dos provadores em relaçãao aos biscoitos amanteigados

Os resultados indicam uma maior aceitabilidade dos biscoitos amanteigados da marca $A$, em que se verifica que $24 \%$ dos provadores certamente comprariam este produto. Já 30\% certamente não comprariam o produto da marca B (Figura 2). 
A definição de perfis sensoriais "ideais" tem sido investigada desde a década de 1970, apesar de esses produtos considerados ditos "ideais" possuírem suas limitações, uma vez que os consumidores divergem uns dos outros nas suas percepções, preferências e atitudes. Além disso, também é importante considerar as diferenças individuais, que acabam ofertando produtos aceitáveis para a maioria dos consumidores, entretanto raramente satisfazem as expectativas do cliente na sua totalidade. Assim, a segmentação com base em classificações hedônicas ou em outras variáveis relacionadas com os consumidores é uma parte vital para a pesquisa com consumidores (SEPPÄ et al., 2013).

A tomada de decisão é uma tarefa comum, que envolve a comparação de diferentes alternativas que são avaliadas de acordo com a prioridade de vários critérios. Normalmente, esses critérios não são independentes e interagem uns com os outros para determinar a decisão final. A análise da relação entre as características visuais e intenção de compra é de interesse para a atitude mais conhecida do consumidor e também para prever-se a intenção de compra ou disposição para pagar (ALFONSO, 2013).

\section{CONCLUSÃo}

Verifica-se, para os testes de reconhecimento de aromas, que os provadores tiveram maior dificuldade de identificar as amostras de pimenta calabresa e coentro, sendo que as maiores percepções sensoriais foram para os aromas das amostras de limão, café, canela, cravo e vinagre. A partir dos resultados observados, foi possível verificar que as práticas realizadas podem contribuir para que 0 aluno exercite sua capacidade de perceber diferenças entre aromas de diferentes amostras de alimento e bebida. Portanto, pode-se verificar a contribuição da análise sensorial como ciência, contribuindo de forma didática no aprendizado dos alunos do curso de Nutrição. Cabe destacar a ampla aplicabilidade da análise sensorial não só para alimentos, mas também como ciência interdisciplinar aplicada para a produção de resultados relevantes.

\section{REFERÊNCIAS}

ADHIKARI, K. et al. Flavor threshold as affected by interaction among three dairy-related flavor compounds. Journal of Sensory Studies, v.21, n.6, p.626-643, Dec. 2006.

ALFONSO, L. A technical note on the use of choquet integral to analyze consumer preferences: application to meat consumption. Journal of Sensory Studies, v.28, p.467-473, 2013.

ALTISENT, R. et al. Injection of flavor essences into fruit pieces: a new approach for exploring consumer preferences for novel flavors of apple fruit. Journal of Sensory Studies, v.28, p.405-413, 2013.

ARAÚJO, W. M. C. et al. Alquimia dos alimentos. Brasília: Editora Senac-DF, 2011. 500p.

ASSOCIAČÃO BRASILEIRA DE NORMAS TÉCNICAS - ABNT. Análise sensorial dos alimentos e bebidas: terminologia. 1993. $8 \mathrm{p}$.
DE MELO LISBOA, H.; PAGE, T.; GUY, C. Gestão de odores: fundamentos do nariz eletrônico. Revista Engenharia Sanitária e Ambiental, v.14 n.1, p.9-18, jan/mar. 2009.

DOTY, R. L.; SHAMAN, P.; DANN, M. Development of the University of Pennsylvania Smell Identification Test: A standardized microencapsulated test of olfactory function. Physiology \& Behavior, v.32, n.3, p.489-502, Marc. 1984.

ELMAN, I.; SILVA, M. E. M. P. Crianças portadoras de leucemia linfóide aguda: análise dos limiares de detecção dos gostos básicos. Revista Brasileira de Cancerologia, v. 53, n. 3, p. 297-303, 2007.

GOYERT, H. et al. Characteristic component odors emerge from mixtures after selective adaptation. Brain Research Bulletin, v.72, n.1, p.1-9, Apr. 2007. 
INSTITUTO ADOLFO LUTZ. Métodos físicoquímicos para análise de alimentos. São Paulo: Instituto Adolfo Lutz, 2008.

KRANC, R.F.R. Olfactory receptors and the mechanism of odor perception. Polish Annals of Medicine, v.20, n.1, p.51-55, Sept. 2013.

MINIM, V. P. R. Análise sensorial: estudos com consumidores. Viçosa: Editora UFV, 2006. 225p.

PARAVISINI, L. et al. Caramel odor: Contribution of volatile compounds according to their odor qualities to caramel typicality.

International, v.57, p.79-88, Marc. 2014.

PEREIRA, R. B., BENNETT, R. J.; LUCKMAN, M. S. Instrumental and sensory evaluation of textural attributes in cheese analogs: a correlation study. Journal of Sensory Studies, v.20, p.434-453, 2005.

SCHUTZ, H.G. A food action rating scale for measuring food acceptance. Journal of Food Science, v.30, n.2, p. 365-374, 1965.

SEPPÄ, L. et al. Hedonic responses and individual definitions of an ideal apple as predictors of choice. Journal of Sensory Studies, v.28, p.346-57, 2013.

SIDEL, J. L.; STONE, H. The role of sensory evaluation in the food industry.
Food Quality and Preference, v.4, n.1-2, p.65-73, 1993.

SIMÕES, D. R. S.; WASZCZYNSKYJ, N.; WOSIACKI, G. Aromas em maçãs, suco e sidra: uma revisão. Boletim do Centro de Pesquisa e Processamento de Alimentos, v.27, n.1, p. 153-172, 2009.

STONE, H.; SIDEL, J. Sensory evaluation practices. Academic Press: New York, 1993. 338p.

STONE, H.; PANGBORN, R. M.; OUGH, C.S. Techniques for sensory evaluation of food odors. Advances in Food Research, v.14, p.1-32, 1965.

TEIXEIRA, L. V. Análise sensorial na indústria de alimentos. Revista do Instituto de Laticínios Cândido Tostes, v. 64, n. 366, p. 12-21, 2009.

TEMPÈRE, S. et al. "Brett character" in wine: Is there a consensus among professional assessors? A perceptual and conceptual approach. Food Quality and Preference, v. 34, p.29-36, Jun. 2014.

TEMPÈRE, S. et al. The training level of experts influences their detection thresholds for key wine compounds. Chemosensory Perception, v.4, p.99$115,2011$.

TRAJANO, V. N. et al. Propriedade antibacteriana de óleos essenciais de especiarias sobre bactérias contaminantes de alimentos. Ciência e Tecnologia de Alimentos, 2009, v.29, n.3, p.542-545. 